\title{
MS06-P08 | Use Of The Cocomaps Web Server To Analyze And Visualize The Interface Of Biomolecular Complexes In Crystal Structures
}

Oliva, Romina (University of Naples "Parthenope", Naples, ITA); Cavallo, Luigi (Kaust Catalysis Center, King Abdullah University of Science and Technology, Thuwal, SAU)

Interactions between biomolecules are at the basis of many of the most important molecular processes in the cell. Therefore, characterizing the interface of biological complexes is a fundamental step for possible biomedical and biotechnological applications and the number of crystal structures solved for them is steadily increasing.

COCOMAPS (bioCOmplexes COntact MAPS) is a user-friendly web application for the analysis, visualization and comparison of the interaction surface in biological complexes, such as protein-protein, protein-DNA and proteinRNA assemblies. It provides information, organized in tables, on the: i) interacting residues, defined on the basis of a cut-off distance, ii) residues at the interface, defined on the basis of the accessible surface area, and iii) intermolecular H-bonds. However, the hallmark of COCOMAPS is the contact map it provides, that is a two-dimensional representation of the interface where a dot is present at the cross-over of two residues which are in contact, i.e. within a cut-off distance. An online 3D visualization of the complex in Jmol and a ready-to-run PyMol script are also provided.

Input files can be downloaded directly from the wwPDB or uploaded locally. More chains can be selected for each interacting partner. Therefore, COCOMAPS can be used to analyze the interface between two molecules, between one molecule and an assembly or between two assemblies, depending on how many chains are specified.

Examples of the use of COCOMAPS for the analysis of crystal structures of biomolecular complexes in the corresponding original papers will be presented and discussed.

COCOMAPS is available at: https://www.molnac.unisa.it/BioTools/cocomaps. 